\title{
Decreased Peripheral Blood Lymphocyte-Monocyte Ratio and Platelet-Monocyte Ratio and the Peripheral Blood Score Model Predict Poor Survival in Peripheral T-cell Lymphoma Patients
}

\section{Yan Zhang}

Zhejiang University Huzhou Hospital: Huzhou Central Hospital

\section{Yuanfei Shi}

Zhejiang University School of Medicine First Affiliated Hospital Huafei Shen

ZheJiang university school of medicine first affiliated hospital Lihong Shou

Zhejiang University Huzhou Hospital: Huzhou Central Hospital

\section{Qiu Fang}

Zhejiang University Huzhou Hospital: Huzhou Central Hospital

\section{Xiaolong Zheng}

Zhejiang University School of Medicine First Affiliated Hospital

\section{Mingyu Zhu}

Zhejiang University school of medicine first affiliated hospital

\section{Xin Huang}

Zhejiang University School of Medicine First Affiliated Hospital

\section{Jiansong Huang}

zhejiang university school of medicine affiliated hospital

\section{Li Li}

zhejiang university school of medicine affiliated hospital

\section{De Zhou}

Zhejiang University School of Medicine First Affiliated Hospital

\section{Lixia Zhu}

Zhejiang University School of Medicine First Affiliated Hospital Jingjing Zhu

Zhejiang University School of Medicine First Affiliated Hospital

\section{Xiujin Ye}

Zhejiang University School of Medicine First Affiliated Hospital Jie Jin

Zhejiang University School of Medicine First Affiliated Hospital 


\section{Wanzhuo Xie ( $\nabla$ xiewanzhuo@zju.edu.cn )}

Zhejiang University School of Medicine First Affiliated Hospital

\section{Research}

Keywords: peripheral T-cell lymphoma, lymphocyte, platelet, monocyte, prognosis, tumor microenvironment, immunity

Posted Date: December 22nd, 2020

DOl: https://doi.org/10.21203/rs.3.rs-130878/v1

License: (c) (i) This work is licensed under a Creative Commons Attribution 4.0 International License. Read Full License 


\section{Abstract}

Background. Peripheral T-cell lymphoma(PTCL) is a group of lymphoproliferative tumors originated from post-thymic T cells or mature natural killer (NK) cells. It shows highly aggressive clinical behaviour, resistance to conventional chemotherapy, and a poor prognosis. Its incidence rate in China is 1 to 2 times higher than in western countries. Therefore, optimal strategies for identifying high-risk patients are urgently needed.

Methods. We retrospectively studied 347 newly diagnosed PTCL patients from January 2011 to October 2019 and analyzed the relationship between peripheral blood lymphocyte-monocyte ratio (LMR) and platelet-monocyte ratio (PMR) and prognosis. The model of Peripheral Blood Score was established to screen out high-risk patients.

Results. The receiver operating characteristic (ROC) curve was used to determine the optimal cut-off value based on survival rate. It was found that patients with PTCL with LMR $\leq 1.68$ and PMR $\leq 300$ had inferior overall survival (OS) and the difference was significant in both low-risk (P凶0.001) and medium high-risk (P凶0.001) groups of IPI score. In multivariate analysis, LMR $\leq 1.68(\mathrm{HR}=1.751,95 \% \mathrm{Cl} 1.158$ 2.647, $p=0.006), P M R \leq 300(H R=1.762,95 \% \mathrm{Cl} 1.201-2.586, p=0.002)$, stage IIIIIV $(H R=3.276,95 \% \mathrm{Cl}$ 1.512-7.099, $p=0.003$ ), Eastern Cooperative Oncology Group (ECOG) score 3-5 (HR=2.351, 95\% Cl 1.647$3.356, \mathrm{p} \otimes 0.001)$ and extra-nodal invasion more than one site ( $\mathrm{HR}=1.659,95 \% \mathrm{Cl} 1.125-2.445, \mathrm{p}=0.039)$ were independently associated with short survival. LMR and PMR were integrated into "Peripheral Blood Score (PBS)" model. PTCL patients were divided into three risk groups: low-risk group, medium risk group and high-risk group. The 1 -year OS was $86 \%, 55.3 \%$ and $22.6 \%$, and the 3 -year OS was $43.4 \%, 20 \%$ and $13.1 \%$, respectively.

Conclusion. Overall, LMR and PMR can be used as early prognostic indicators in PTCL patients. Moreover, we can easily detect the complete blood cell count (CBC), and use PBS model to preliminarily screen and stratify patients. It is simple, convenient and accurate to screen out patients with short lives, and formulate personalized treatment strategies.

\section{Background}

Peripheral T-cell lymphoma(PTCL) is a group of rare hematological malignancies with heterogeneous morphological and biological characteristics. The overall manifestations are high invasiveness, short survival and poor prognosis. The total incidence rate is $0.5-2$, per 100000 persons per year, and account for about $10 \%$ of all non-Hodgkin's lymphomas (NHL) in Western countries[1]. However, the incidence of PTCL in Asia is higher, accounting for $25 \%-30 \%$ of NHL in China[2]. Nowadays, the internationally recommended first line therapy is still anthracycline-based chemotherapy. But the complete response (CR) rate after chemotherapy is only $40 \%-60 \%$, with overall survival (OS) of $30-40 \%$. Most patients face the problem of short-term recurrence [3]. And the recurrence or progression of the lack of effective treatment measures [4-5]. 
According to the WHO classification, PTCL can be further divided into many pathological subtypes. The most common subtypes include PTCL-not otherwise specified (PTCL-NOS), extra-nodal natural killer (NK)/T cell lymphoma, nasal type (ENKTL), angioimmunoblastic T-cell lymphoma (AITL), anaplastic lymphoma kinase positive anaplastic large cell lymphoma (ALK+ ALCL) and anaplastic lymphoma kinase negative anaplastic large cell lymphoma (ALK- ALCL) [6]. In addition, there are some rare subtypes, such as monomorphic epitheliotropic intestinal T-cell lymphoma(MEITL), subcutaneous panniculitis like T-cell lymphoma(SPTCL), mycosisfungoides/Sezary's syndrome(MF/SS), Hepatosplenic T-cell lymphoma (HSTCL) and so on. Due to its rarity and heterogeneity, the prognosis of PTCL are less studied.

International Prognostic Index (IPI) confirmed its usefulness for PTCL including age, Eastern Cooperative Oncology Group (ECOG), Ann Arbor staging, lactic dehydrogenase (LDH) and extra-nodal invasion [3]. The Intergruppo Italiano Linfomi (now Fondazione Italiana linfomi, FIL) performed a large study in the 1990s on 385 patients diagnosed and treated. Based on this study, they identified a prognostic model, the Prognostic Index for PTCL-unspecified (PIT). The results showed that age, ECOG, LDH and bone-marrow involvement were independent predictors of OS and they confirmed that the PIT was slightly more effective than the IPI in PTCL-NOS patients[7]. But even so, these scoring systems are relatively complex and need to be evaluated from multiple aspects. Therefore, our study found that through the determination of peripheral blood lymphocyte-monocyte count ratio and platelet-monocyte count ratio could be simple and effective to screen out high-risk patients.

Tumor micro-environment, host immunity and systemic inflammatory response are the key factors that determine the clinical course and prognosis of tumor patients. More and more studies found tumorassociated macrophages (TAMs) are associated with poor clinical prognosis of a variety of tumors, including colon cancer, thyroid cancer and Burkitt's lymphoma by promoting angiogenesis, local invasion and metastasis[8-10]. Moreover, it has been confirmed that the growth and survival of malignant $\mathrm{T}$ cells depend on alternatively activated (M2) macrophages in micro-environment, and the growth of alternatively activated (M2) macrophages are affected by TAMs [11-12]. Yingming Zhu et al. found that serum monocytes were locally absorbed and differentiated into macrophages after tumor invasion, and reacted to a wide range of chemokines and growth or differentiation factors such as CCL-2 and CSF-1 produced by tumor and stromal cells, and further confirmed the correlation between LMR and TILs / TAMs ratio[13].

Increasing evidence indicates that platelets play several roles in the progression of malignancies and in cancer-associated thrombosis. A notable cross-communication exists between platelets and cancer cells[14]. CXCL12 promoted monocytes and M1-M2 macrophages to phagocytic apoptosis platelets, and promoted their differentiation into foam cells through CXCR4 and CXCR7. Therefore, platelet derived CXCL12 could regulate monocyte macrophage functions through the different binding of CXCR4 and CXCR7, which indicated that they played an important role in the inflammatory reaction of platelet aggregation site[15]. Our study is the first time to discuss the relationship between PMR, LMR and prognosis of PTCL. We found that the decreased of PMR and LMR were closely related to the low survival 
of PTCL. On this basis, we established a peripheral blood score (PBS) model to identify high-risk patients early.

\section{Materials And Methods}

\subsection{Patients and characteristics}

This is a single center retrospective study. A total of 347 patients with PTCL newly diagnosed in the First Affiliated Hospital of Zhejiang University School of Medicine from January 2011 to October 2019 were included. The final observation time was January 2020, and the median follow-up time was 18 months (rang: 0-108 months). The inclusion criteria were as follows: (1) Age $\geq 15$ years; (2) The pathological diagnosis was consistent with PTCL; (3) Newly diagnosed and no chemotherapy before clinical data were collected; (4) Complete clinical data; (5) At least two cycles of treatment were given. Although the treatment plan were not completely unified, all the patients in our study received cyclophosphamidedoxorubicin-vincristine-prednisone (CHOP) or CHOP-like chemotherapy regimen, and all ENKTL patients were treated with chemotherapy combined with Pegaspargase. All procedures involving human participants in our study were conducted in accordance with the Helsinki declaration.

We collected the medical records, physical examinations, laboratory results, pathological reports and radiological results of these patients through electronic medical records, and re analyzed the clinical data of them. All baseline data are presented in Table 1. Follow-up was performed by making phone calls. The absolute counts of lymphocytes, monocytes and platelets were obtained from a standard complete blood count $(\mathrm{CBC})$ performed at diagnosis. LMR was the absolute count of lymphocyte divided by the absolute count of monocyte. PMR was the ratio of platelet absolute count to monocyte absolute count. OS was defined as the time from diagnosis to death for any reasons or last follow-up.

\subsection{Cut-off value for LMR/PMR}

The optimal cut-off of LMR and PMR were obtained by calculating the area under the curve by receiver operating characteristic (ROC) curve analysis.

\subsection{Statistical analysis}

Post hoc power analyses were conducted with GPOWER (Faul, Erdfelder, Lang, \& Buchner, 2007) in order to estimate the probability of occurrence of effects in the sample. Through the normal distribution test, the continuous variables included in this study all conform to the normal distribution. The continuous variables such as lymphocyte count, monocyte count and platelet count were shown as median with range and were compared by Mann-Whitney U-test. Other continuous variables were grouped according to the usual clinical threshold and were presented as frequencies and percentages $(n, \%)$ in company with categorical variables. All hierarchical and categorical variables were compared by Pearson's chi-square test. Among them, histological subtypes were performed bonferroni-post-hoc-correction. Kaplan-Meier curve was used to analyze OS and log-rank test was used for comparison. Univariate and multivariate 
logistic regression models were used to evaluate the correlation between clinical variables and complete remission (CR). Cox proportional hazard regression model was used to analyze univariate and multivariate of OS. Statistical analysis was performed by spss23.0 software package. In all comparisons, the results were considered to be statistically significant when the $p$ value was $<0.05$, and $95 \%$ confidence interval $(\mathrm{Cl})$ was given.

\section{Results}

\subsection{Patients Characteristics}

According to the admission conditions, we enrolled 347 patients with newly diagnosed PTCL for analysis. The clinical characteristics and laboratory data were listed in Table 1. The four most common subtypes of PTCL-NOS, ENKTL, AITL and ALCL accounted for $32.0 \%, 32.5 \%, 20.2 \%$ and $11.8 \%$, respectively. Other rare subtypes including MEITL, SPTCL, HSTCL and MF/SS accounted for $3.5 \%$ totally. However, the different subtypes of PTCL did not show statistical significance in the stratification of LMR in our study, either by Pearson's chi square test $(P=0.330)$ or performing bonferroni-post-hoc-correction $(P=0.570)$. Although the difference of case typing in PMR was statistically significant through Pearson's chi square test $(P=0.018)$, the difference was not statistically significant in bonferroni-post-hoc-correction $(P=0.139)$. The median age at diagnosis was 55 years (rang: $15-84$ years), and among them, $36 \%$ of the elderly patients were over 60 years old. The ratio of male to female was close to $2: 1$. About $81.3 \%$ of patients were in stage III-IV and $58.5 \%$ of them had B symptoms. At the first diagnosis, $32.3 \%$ of patients had albumin below $35 \mathrm{~g} / \mathrm{L}$, and $64.6 \%$ of them were infected with Epstein-Barr virus (EBV). In addition, 163 patients had more than one extra-nodal site involved. The serum lactic dehydrogenase (LDH) and beta-2 microglobulin ( $\beta 2-\mathrm{MG}$ ) levels were increased in $64 \%$ of the patients, respectively.

All patients received at least two cycles of chemotherapy. Except for all ENKTL patients received the protocol containing Pegaspargase. The rest of patients included in this study were treated with $\mathrm{CHOP}$ (cyclophosphamide-doxorubicin-vincristine-prednisone) or CHOP-like chemotherapy. Furthermore, 36 patients proceeded to hemopoietic stem cell transplantation, in which 24 of them accepted autologous stem cell transplantation.

3.2 The cut-off values and the association of LMR/PMR with clinical parameters and complete response (CR)

The ROC curve was generated to select the appropriate cutoff values for LMR and PMR based on the survival analysis (Figure 1). For LMR, the area under curve (AUC) was 0.734 (95\% Cl: 0.682-0.786), with a generated maximum joint sensitivity and specificity at the value of 1.68. In addition, for PMR the AUC was calculated to be 0.718 (95\% Cl: $0.664-0.772)$, with a generated maximum joint sensitivity and specificity at the value of 300 . Table 1 compared the clinical characteristics of patients with LMR and PMR at different levels and the high group and low group were defined as being greater than the cutoff value and less or equal to than the cutoff value, respectively. Post hoc analysis demonstrated sufficient power to distinguish the significant differences (power $=0.996$ ). Patients with LMR $\leq 1.68$ or PMR $\leq 300$, 
only $14.2 \%$ and $14.3 \%$ of them achieved complete response (CR) after treatment. Therefore, it is not difficult to speculate that patients with $L M R \leq 1.68$ or $P M R \leq 300$ may have poor therapeutic effect. Moreover, we found that in the low LMR and PMR groups, the proportion of patients with IPI at 3-5 points is higher. Whether in the low-risk group (IPI = 0-2) or the high-risk group of IPI (IPI = 3-5), the OS of PTCL patients with low LMR group and low PMR group were significantly lower than those of patients in the high LMR group and high PMR group (Figure 3).

In univariate logistic regression analysis, lower $\mathrm{CR}$ rate was related to first diagnosis older than 60 years, $I P I \geq 3, E C O G \geq 3$, stage III-IV, B symptoms, bone marrow involvement, Albumin $\varangle 35 \mathrm{~g} / \mathrm{L}$, EBV infection, Extra-

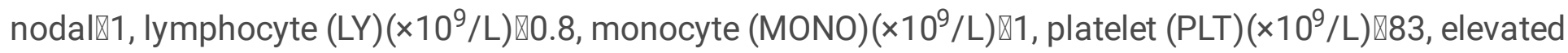
$L D H$ and elevated $\beta 2-M G, L M R \leq 1.68$ and $P M R \leq 300$ (Table 2). Nevertheless, in the multivariate logistic regression analysis, only first diagnosis older than 60 years $(\mathrm{OR}=4.031,95 \% \mathrm{Cl} 2.021-8.041$, $\mathrm{p} \otimes 0.001)$, $E C O G \geq 3(O R=3.610,95 \% \mathrm{Cl} 1.572-8.290, p=0.002)$, stage IIIIIV(OR=2.737, 95\% Cl 1.255-5.969, $p=0.011)$, bone marrow involvement(OR=2.581, 95\% $\mathrm{Cl} 1.173-5.683, \mathrm{p}=0.018)$ and EBV infection(OR=2.090, $95 \% \mathrm{Cl}$ 1.170-3.734, $p=0.013$ ) were statistically significant(Table 2 ).

\subsection{The association of LMR/PMR with OS}

In our study, we analyzed the survival of low and high groups of LMR and PMR patients, and found that there were significant differences in OS between all the two groups (Pख0.001)(Figure 2). The median survival time in the groups with $L M R \leq 1.68$ and $L M R \otimes 1.68$ and $P M R \leq 300$ and $P M R \bowtie 300$ were 5 months and 28.5 months, 6 months and 28 months, respectively. The univariate analysis showed that first diagnosis older than 60 years, IPI $\geq 3, E C O G \geq 3$, stage III-IV, B symptoms, bone marrow involvement,

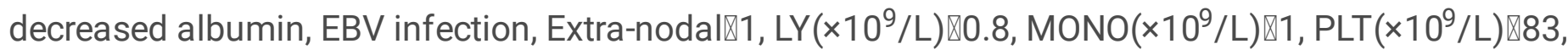
elevated LDH, elevated $\beta 2-M G, L M R \leq 1.68$ and $P M R \leq 300$ were prognostic indicators of OS(Table 3 ). Then, multivariate analysis was showed that patients with poor OS had ECOG $\geq 3(\mathrm{HR}=2.351,95 \% \mathrm{Cl}$ 1.647-3.356, p $₫ 0.001)$, stage IIIIIV (HR=3.276, 95\% Cl 1.512-7.099, $p=0.003)$, Extra-nodal $ه 1$ (HR=1.659, $95 \% \mathrm{Cl} 1.125-2.445, \mathrm{p}=0.039), \mathrm{LMR} \leq 1.68(\mathrm{HR}=1.751,95 \% \mathrm{Cl} 1.158-2.647, \mathrm{p}=0.006)$, and $\mathrm{PMR} \leq 300$ (HR=1.762, 95\% Cl 1.201-2.586, $\mathrm{p}=0.002)$.

Considering the heterogeneity of PTCL, we analyzed the five common pathological subtypes separately, and found that the decrease of LMR and PMR was significantly correlated with OS except ALCL, ALK+ (Table 4, 5). May be related to the small number of this subtypes in this study.

\subsection{Establishment of PBS model and its correlation with OS}

We compared the peripheral blood cell count of the patients at the initial stage, and scored 1 point for $L M R \leq 1.68$ and PMR $\leq 300$ respectively. The patients were divided into PBS 0,1 and 2.0 was divided into low-risk group, 1 was medium risk group, and 2 was high-risk group. The OS of the three groups was statistically analyzed by Kaplan Meier curve and log-rank test. It was found that the OS of low-risk, medium risk and high-risk patients was significantly different. The median OS of the three groups were 32.5 months, 13 months and 5 months respectively (P凶0.001)(Figure 4). Not only that, in the PBS high- 
risk group, $77.4 \%$ of the patients survived for less than 1 year, and only $13.1 \%$ survived for more than 3 years. In the low-risk group with PBS 0 score, $86 \%$ of the patients survived more than 1 year, and $43.4 \%$ of the patients survived for more than 3 years, nearly half of them.

\section{Discussion}

The prognosis of PTCL is poor, either in first-and second-line therapy or salvage therapy. There is an urgent need to use accurate predictive models to classify patients at risk. Among all the previously reported indices, IPI and PIT are the most commonly used. It is worth noting that there is considerable overlap in the parameters used to establish the various models and that the patients need to be evaluated by imaging, examination, bone marrow, etc. . The operations are complex, and the establishments of these scoring models are not entirely based on PTCL, so their accuracy are questionable.

With the development of medical science in recent years, more and more attention had been paid to the study of tumor molecular mechanisms, especially in tumor micro-environment. Therefore, the research on PTCL has opened a new chapter.

Recently, a growing body of research has consistently shown that tumor-associated inflammatory response is a key determinant of prognosis in cancer patients [16]. In previous retrospective studies, we found an association between increased serum Interleukin-10 levels and low survival and early recurrence in patients with PTCL [17]. Interleukin-10 is involved in the body's inflammatory and immune responses and plays an important role in tumors and infections. It is mainly secreted by monocytes, activated Tcells, macrophages, certain tumor cells and so on. Thus, monocyte and macrophage systems are closely related to the prognosis of PTCL. About 2-9\% of peripheral leukocytes are peripheral blood monocytes (PBMC), but only $40 \%$ of them are used for monocyte circulation, while $60 \%$ of monocytes migrate [18]. However, some immature PBMC can differentiate into specialized, tissue-specific macrophages and Antigen-presenting cells (APC). Their differentiation directly determines their functions. The differentiated monocytes/macrophages (Mphi) plays a specific role in the cell mediated innate immunity against infection, immunoregulation, morphogenetic remodelling and malignancy or tissue repair [19-20]. Subsequently, macrophages respond to a wide range of chemokines and growth or differentiation factors such as CCL-2 and CSF-1 produced by tumors and stromal cells. VEGFA and EGF produced by Tumorassociated macrophages (TAMs) promote angiogenesis and tumor growth, respectively, which is consistent with the positive correlation between CD68 expression, vascular invasion and tumor length [21]. Macrophages are remarkably plastic. Depending on the stimulus that activates them, they can polarize to either type M1 (causing an anti-tumor response) or type M2 (causing tumor growth and progression). It had been demonstrated that the growth and survival of malignant T-cells depend on alternatively activated (M2) macrophages in the micro-environment and that the growth of alternatively activated (M2) macrophages were influenced by TAMs [22]. Yingming Zhu et al. found that LMR was associated with TILs/TAMs ratio, and that low LMR had worse OS in patients with esophageal squamous cell carcinoma [13]. It suggests that a systemic inflammatory response may reflect concurrent focal inflammation in the tumor. 
D.lacono et al. retrospectively analyzed 165 patients with advanced melanoma. The severity and prognosis of the disease were assessed. The decrease of LMR suggests short OS and more distant metastatic sites in malignant melanoma [23]. Wang et al. reported 355 cases of diffuse large B-cell lymphoma (DLBCL). In the low LMR group, PFS and OS were shorter and M2-TAM content was higher. These results suggested that weak anti-tumor immunity may be an adverse prognostic factor for aggressive lymphoma, identified in high-risk patients [24]. Thus, lymphocyte counts, monocyte counts, and LMR surrogate markers of tumor micro-environment had been reported as prognostic factors for $B$ cell lymphoma[24-26]. Similarly, recent studies had shown that both lymphocyte counts and monocyte counts could predict the clinical outcome of T-cell lymphomas[27-28]. And patients with T-cell lymphomas after autologous peripheral hematopoietic stem cell transplantation had longer OS and PFS with autograft lymphocyte-to-monocyte ratio(A-LMR) greater than or equal to 1. Compared with patients with A-LMR less than 1, the five years OS rate was $87 \%$ to $26 \%$, and the five years PFS rate was $72 \%$ to $16 \%$, significant difference [29]. But in our study, patients with LMR less than or equal to 1.68 had a median OS and significantly worse CR rate for treatment than patients with LMR greater than 1.68 (5 months vs. 28.5 months; $14.2 \%$ vs. $45 \%$ ). The main reasons for this difference include: first, in Luis $\mathrm{F}$ et al.'s study, they examined autograft lymphocyte-to-monocyte ratio(A-LMR), whereas our study examined LMR in patients'peripheral blood at the time of initial diagnosis; second, they included only 109 patients, we included 347 patients; third, they chose patients with T- cell lymphomas, and we included peripheral Tcell lymphomas, with different baseline characteristics.

Megakaryocytogenesis is a process in which megakaryocytes (MK) proliferate, differentiate and mature from pluripotent hematopoietic stem cells (HSC), while platelets are formed from mature MK fragments. The average platelet count in humans is between $150 \times 10^{9}$ and $400 \times 10^{9}$ per liter, but over time, the number of individual platelets remains the same[30]. Platelets mainly participates in the organism haemostasis and the thrombosis. In recent years, there are increasing evidences that platelets and tumor cells have significant cross-communication, suggesting that they play an important role in the progression of malignant tumors, the occurrence of tumor-associated local inflammation, and cancerassociated thrombosis. On the one hand, tumors can affect the RNA profile of platelets, the number of circulating platelets and their activation status. On the other hand, tumor-induced platelets contain a large number of active biomolecules, including platelet-specific and circularly ingested biomolecules that are released upon activation of platelets and are involved in the development of malignant tumors[31]. Tissue factor (TF) has a direct pro-inflammatory effect by inducing the production of reactive oxygen species [32]. Thus, platelets are directly involved in the initiation of inflammatory responses (clotting, monocyte recruitment, activation, and matrix remodeling) by inducing additional adhesion molecules, MMPs, tissue-type plasminogen activators, and cytokines (such as MCP-1) in endothelial cells [33]. Platelets store and release CXCL12 (SDF-1), which controls the differentiation of hematopoietic progenitors into endothelial cells or macrophage-foam cells. CXCL12 binds CXCR4 and CXCR7 and regulates the functions of monocyte/macrophages. $M$ Chatterjee et al. found that platelets and plateletmacrophage aggregates increased in peritoneal fluid following peritonitis induction in mice in vivo. Compared with peripheral blood, the relative surface expressions of CXCL12, CXCR4 and CXCR7 in 
infiltrated monocytes were also enhanced. Moreover, platelet CXCL12 and recombinant CXCL12 participate in the enhancement of specific induction of monocyte chemotaxis through CXCR4. Under static and dynamic arterial flow conditions, the adhesion of monocytes to the surface of immobilized CXCL12 and activated platelets rich in CXCL12 is mediated mainly through CXCR7, and is counterregulated by neutralizing platelet-derived $\mathrm{CXCL} 12$. In the co-culture experiment with platelets, monocytes were mainly differentiated to CD163+ macrophages, and CD163+ macrophages weakened after blocking antibodies to CXCL12 and CXCR4/CXCR7. Therefore, platelets-derived CXCL12 can regulate mononuclear-macrophage functions through different combinations of CXCR4 and CXCR7, suggesting that it plays an important role in platelet aggregation in local inflammatory responses [34].

Platelet activation plays an important role in tumor-associated immune thrombosis and multiple metastasis. Activated platelets were known to secrete a range of inflammatory chemokines that activate inflammatory signaling pathways in white blood cells, including PAF, RANTES, CCL3, CXCL1, CXCL4 (platelet factor 4), and CXCL7 [35, 36]. Serotonin (5-hydroxytryptamine) is another platelet-releasing product that may affect monocyte function. Monocytes exposed to 5-hydroxytryptamine showed increased NF-KB activation, increased cytokine production induced by LPS, and decreased apoptosis, possibly due to changes in BCL-2 or MCL-1 expression [37]. In the past few decades, a large number of clinical studies have shown that daily aspirin can reduce the incidence, metastasis and mortality of tumors, especially for colorectal cancer [38]. Recently, Guillem Lobat P and his collaborators demonstrated in an immunodeficiency mouse model that low-dose aspirin reduces the metastasis of lung cancer by avoiding the enhanced pro-aggregation effect caused by platelet-tumor cell interaction [39]. These clinical studies have fully confirmed that platelets are closely related to the occurrence and development of tumors.

In conclusion, since both host immunity and tumor micro-environment are closely related to the occurrence, development and metastasis of tumor, the combination of lymphocyte count, monocyte count and platelet count can better reflect the prognosis of tumor. LMR had been studied in many different disease settings, had been widely discussed, and had been found to predict esophageal cancer, melanoma, Hodgkin's lymphoma, multiple myeloma, and DLBCL [13,23,40-42]. However, it has never been discussed in PTCL. Platelet counts and monocyte counts had been shown to be associated with sepsis, pulmonary embolism [43-44], and so on, but no correlation between platelet counts and monocyte count ratios and tumors has been previously reported. We analyzed 347 patients with primary PTCL and found that the patients with low LMR ratio and low PMR ratio had worse response to treatment and shorter survival time. The difference were significant in low-to-moderate-risk patients with an IPI score of 0-2 and in high-to-moderate-risk patients with an IPI score of 3-5. In multivariate tests, LMR $\leq 1.68$ and PMR $\leq$ 300 were independent risk factors for OS shortening. By integrating reduced LMR and PMR to produce a "PBS" model, newly diagnosed PTCL patients can be divided into three risk groups. Specifically, patients in the low-risk group had no abnormal blood cells and had a 3 -year survival rate of $43.4 \%$, while those in the high-risk group had a 3-year survival rate of $13.1 \%$. 
Our study still has some limitations. Firstly, the retrospective study may be biased in the selection of patients. Secondly, the dynamic changes of patients during treatment did not taken into account during analysis. Third, our sample size is relatively small and lack of cytogenetic data. Another issue is the cutoff value of LMR/PMR used in clinical practice. In the past and in our study, the ROC curve based on survival was used to determine the optimal cut-off value, indicating that there was inconsistency between the centers. Therefore, further exploration and prospective trials with larger samples are needed in the future and the PBS model we established also needs further verification.

\section{Conclusions}

All in all, decreased LMR and PMR can be used as early prognostic indicators in PTCL patients. Moreover, we can easily detect the complete blood cell count (CBC), and use PBS model to preliminarily screen and stratify patients. It is simple, convenient and accurate to screen out patients with short lives, and formulate personalized treatment strategies. More large-sample prospective clinical trials are needed to confirm these findings in future research directions.

\section{List Of Abbreviations}

PTCL: Peripheral T-cell lymphoma

LMR: lymphocyte-monocyte ratio

PMR: platelet-monocyte ratio

LY: lymphocyte

MONO: monocyte

PLT: platelet

ROC: receiver operating characteristic

OS: overall survival

CR: complete response

IPI: International Prognostic Index

ECOG: Eastern Cooperative Oncology Group

PBS: Peripheral Blood Score

CBC: complete blood cell count

NHL: non-Hodgkin's lymphomas 
PTCL-NOS: PTCL-not otherwise specified

ENKTL: extra-nodal natural killer (NK)/T cell lymphoma, nasal type

AITL: angioimmunoblastic T-cell lymphoma

ALK+ ALCL: anaplastic lymphoma kinase positive anaplastic large cell lymphoma

ALK- ALCL: anaplastic lymphoma kinase negative anaplastic large cell lymphoma

MEITL: monomorphic epitheliotropic intestinal T-cell lymphoma

SPTCL: subcutaneous panniculitis like T-cell lymphoma

MF/SS: mycosisfungoides/Sezary's syndrome

HSTCL: Hepatosplenic T-cell lymphoma

LDH: lactic dehydrogenase

B2-MG: beta-2 microglobulin

TAMs: tumor-associated macrophages

APC: Antigen-presenting cells

Mphi: monocytes/macrophages

\section{Declarations}

- Ethics approval and consent to participate

All procedures involving human participants in our study were conducted in accordance with the Helsinki declaration. This study was approved by the ethics committee and all patients signed informed consents.

- Consent for publication

This paper has been approved for publication.

- Availability of data and material

This was a retrospective study, all the data of patients were detected in clinical treatment, which were true and effective.

7.4 Competing interests

There is no conflict of interest between the authors. 
- Funding

No funding support.

- Authors' contributions

XW designed the study. ZY, SY, SF, SH, FQ, ZX, ZM, JJ and YX collected the patients' material. HX and HJ checked the test results. ZY, LL, ZD, ZJ and ZL analyzed data. ZY and SY wrote the paper.

\subsection{Acknowledgements}

The authors thank the practitioners who helped to collect and sort out the patient' information and followup and thank the patients for allowing us to analyze their data.

\section{References}

1. A clinical evaluation of the Inernational Lymphoma Study Group classification of non-Hodgkin's lymphoma. The Non-Hodgkin's Lymphoma Classification Project. Blood 1997 Jun 1;89(11): 3909-39

2. Shi Y. Current status and progress of lymphoma management in China.Int J Hematol. 2018; 107(4): 405-

3. D. Weisenburger, K.J. Savage, N.L. Harris, R.D. Gascoyne, E.S. Jaffe, K.A. MacLennan, T. Rudiger, S. Pileri, S. Nakamura, B. Nathwani, E. Campo, F. Berger, B. Coiffier, W.S. Kim, H. Holte, M. Federico, W.Y. Au, K. Tobinai, J.O. Armitage, J.M. Vose, Peripheral T-cell lymphoma, not otherwise specified: a report of 340 cases from the International Peripheral T-cell Lymphoma Project, Blood 117 (2011) 34023408.

4. Bellei, F.M. Foss, A.R. Shustov, S.M. Horwitz, L. Marcheselli, W.S. Kim, M.E. Cabrera, I. Dlouhy, A. Nagler, R.H. Advani, E.A. Pesce, Y.H. Ko, V. Martinez, S. Montoto, C. Chiattone, A. Moskowitz, M. Spina, I. Biasoli, M. Manni, M. Federico, The outcome of peripheral T-cell lymphoma patients failing first line therapy: a report from the prospective, international T-Cell project, Haematologica 103 (2018) 11911197.

5. Fabbri, E. Cencini, A. Pietrini, A. Gozzetti, M. Defina, G. Fontanelli, M.A. Mazzei, L. Volterrani, M. Bocchia, Impressive activity of lenalidomide monotherapy in refractory angioimmunoblastic T-cell lymphoma: report of a case with long-term follow-up, Hematol. Oncol. 31 (2013) 213-217.

6. Iqbal J, Wright G, Wang C. Gene expression signatures delineate biological and prognostic subgroups in peripheral T-cell lymphoma. Blood (2014) 123:2915- doi: 10.1182/blood-2013-11-536359

7. Gallamini, A, Stelitano, C., Calvi, R, Bellei, M., Mattei, D, Vitolo, U, Morabito, F, Martelli, M, Brusamolino, E, lannitto, E, Zaja, F, Cortelazzo, S, Rigacci, L, Devizzi, L, Todeschini, G, Santini, G, Brugiatelli, M. \& Federico, M. Peripheral T-cell lymphoma unspecified (PTCL-U): a new prognostic model from a retrospective multicentric clinical study. Blood, 103(2004), 2474-

8. Alberto Malesci, Paolo Bianchi, Giuseppe Celesti, Gianluca Basso, Federica Marchesi, Fabio Grizzi, Giuseppe Di Caro, Tommaso Cavalleri, Lorenza Rimassa, Richard Palmqvist, Alessandro Lugli, Viktor 
H Koelzer, Massimo Roncalli, Alberto Mantovani, Shuji Ogino, Luigi Laghi. Tumor-associated macrophages and response to 5-fluorouracil adjuvant therapy in stage III colorectal cancer. Oncoimmunology. 2017 Jul 12;6(12):e1342918.doi: 1080/2162402X. 2017.1342918.

9. Mabel Ryder, Ronald A Ghossein, Julio C M Ricarte-Filho, Jeffrey A Knauf, James A Fagin. Increased density of tumor-associated macrophages is associated with decreased survival in advanced thyroid cancer. Endocr Relat Cancer.2008 Dec;15(4):1069-74. doi: 10.1677/ERC-08-0036.

10. Godfred Futagbi, Ben Gyan, Harriet Nunoo, John K A Tetteh, Jennifer E Welbeck, Lorna Awo Renner, Michael Ofori, Daniel Dodoo, Dominic A Edoh, Bartholomew D Akanmori. High Levels of IL-10 and CD4+CD25hi+ Treg Cells in Endemic Burkitt's Lymphoma Patients.2015 Aug 4;3(3):224-236.doi: 10.3390/biomedicines3030224.

11. Xuesong Wu, Brian C Schulte, Youwen Zhou, Dipica Haribhai, Alexander C Mackinnon, Jose A Plaza, Calvin B Williams, Sam T Hwang. Depletion of M2-like tumor-associated macrophages delays cutaneous T-cell lymphoma development in vivo. J Invest Dermatol. J Invest Dermatol.2014 Nov;134(11):2814-2822. doi: 10.1038/jid.2014.206.

12. Mantovani, F. Marchesi, A. Malesci, L. Laghi, P. Allavena. Tumour-associated macrophages as treatment targets in oncology. Nat. Rev. Clin. Oncol. 2017 Jul;14(7):399-416. doi: 10.1038/nrclinonc.2016.217.

13. Yingming Zhu, Minghuan Li, Cong Bo, Xuemei Liu, Jianbo Zhang, Zhenxiang Li, Fen Zhao, Li Kong, Jinming Yu. Prognostic significance of the lymphocyte-to-monocyte ratio and the tumor-infiltrating lymphocyte to tumor-associated macrophage ratio in patients with stage T3NOMO esophageal squamous cell carcinom Cancer Immunol Immunother.2017 Mar; 66(3): 343-354. doi: 10.1007/s00262-016-1931-5.

14. Léa Plantureux, Diane Mège, Lydie Crescence, Françoise Dignat-George, Christophe Dubois and Laurence Panicot-Dubois. Impacts of Cancer on Platelet Production, Activation and Education and Mechanisms of Cancer-Associated Thrombosis. Cancers 2018, 10, 441; doi:10.3390/cancers10110441.

15. M Chatterjee, SNI von Ungern-Sternberg, P Seizer, F Schlegel, M Büttcher, NA Sindhu, S Müller, A Mack and $M$ Gawaz. Platelet-derived CXCL12 regulates monocyte function, survival, differentiation into macrophages and foam cells through differential involvement of CXCR4-CXCR7. Cell Death and Disease (2015) 6, e1989; doi:10.1038/cddis.2015.233.

16. Raina, V., Singhal, M.K., Sharma, A., Kumar, L., Kumar, R., Duttagupta, S., Kumar, B. \& Das, P. Clinical characteristics, prognostic factors, and treatment outcomes of 139 patients of peripheral T-cell lymphomas from AllMS, New Delhi, India. Journal of Clinical Oncology, 2010(28), e18549-e18549.

17. Yan Zhang, Yanlong Zheng, Lihong Shou, Yuanfei Shi, Huafei Shen, Mingyu Zhu, Xiujin Ye, Jie Jin, Wanzhuo Xie. Increased serum level of interleukin-10 predicts poor survival and early recurrence in patients with peripheral T-cell lymphomas. Frontiers in Oncology. 2020 Oct 13; 10:584261. doi:10.3389/fonc. 2020. 584261. 
18. Stefan Reuter, Detlef Lang.Life span of monocytes and platelets: importance of interactions. Frontiers in Bioscience 14, 2432-2447, January 1, 2009.

19. MacDonald KP, Munster DJ, Clark GJ, Dzionek A, Schmitz J, Hart DN. Characterization of human blood dendritic cell subsets. Blood 2002(100), 4512-4520.

20. Steinman RM, Banchereau J. Taking dendritic cells into medicine. Nature 2007(449), 419-426.

21. Noy R, Pollard JW. Tumor-associated macrophages: from mechanisms to therapy. Immunity 41(1):49- doi:10.1016/j. immuni.2014.06.010.

22. Wu X, Schulte BC, Zhou Y. Depletion of M2-like tumor-associated macrophages delays cutaneous Tcell lymphoma development in vivo. J Invest Dermatol. 2014;134:2814-2822.10.1038/jid.2014.206.

23. Iacono, D. Basile, L. Gerratana, M.G. Vitale, G. Pelizzari , M. Cinausero, E. Poletto, F. Puglisi , G. Fasola , A.M. Minisini. Prognostic role of disease extent and lymphocyte-monocyte ratio in advanced melanoma. Annals of Oncology 28 (Supplement 6): vi68vi69, 2017 doi:10.1093/annonc/mdx428.

24. Wang, K. Gao, W. Lei, L. Dong, Q. Xuan, M. Feng, J. Wang, X. Ye, T. Jin, Z. Zhang, Q. Zhang. Lymphocyte-to-monocyte ratio is associated with prognosis of diffuse large B-cell lymphoma: correlation with CD163 positive M2 type tumor-associated macrophages, not PD-1 positive tumorinfiltrating lymphocytes, Oncotarget 8 (2017) 5414-5425.

25. Kumagai S, Tashima M, Fujikawa J, Iwasaki M, Iwamoto Y, Sueki Y, et al. Ratio of peripheral blood absolute lymphocyte count to absolute monocyte count at diagnosis is associated with progressionfree survival in follicular lymphoma. Int J Hematol. 2014;99:737-

26. Wilcox RA, Ristow K, Habermann TM, Inwards DJ, Micallef IN, Johnston PB. The absolute monocyte and lymphocyte prognostic score predicts survival and identifies high risk patients in diffuse large Bcell lymphoma. Leukemia. 2011;25(9):1502-

27. Huang JJ, Li YJ, Xia Y, Wang Y, Wei WX, Zhu YJ. Prognostic significance of peripheral monocyte count in patients with extranodal natural killer/T cell lymphoma. BMC Cancer. 2013;13:222.

28. Mitrovic Z, Perry AM, Suzumiya J, Armitage JO, Au WY, Coiffier B, et al. The prognostic significance of lymphopenia in peripheral $T$ cell and natural killer/T cell lymphomas: a study of 826 cases from the International Peripheral T cell lymphoma project. Am J Hematol. 2012;87(8):790-

29. Luis F. Porrata, David J. Inwards, Stephen M. Ansell, Ivana N. Micallef, Patrick B.Johnston, William J. Hogan and Svetomir N. Markovic. Infused autograft lymphocyte-to-monocyte ratio and survival in Tcell lymphoma post autologous peripheral blood hematopoietic stem cell transplantation. Journal of Hematology \& Oncology,2015,DOI 10.1186/s13045-015-0178-5.

30. Harker LA, Roskos LK, Marzec UM, Carter RA, Cherry JK, Sundell B, Cheung EN, Terry D, Sheridan W. Effects of megakaryocyte growth and development factor on platelet production, platelet life span, and platelet function in healthy human volunteers. Blood 95, 2514-2522 (2000).

31. Léa Plantureux, Diane Mège, Lydie Crescence, Françoise Dignat-George, Christophe Dubois and Laurence Panicot-Dubois.Impacts of Cancer on Platelet Production, Activation and Education and Mechanisms of Cancer-Associated Thrombosis.Cancers 2018, 10, 441; doi:10.3390/cancers 10110441 . 
32. Herkert O, Diebold I, Brandes RP, Hess J, Busse R, Gorlach A. NADPH oxidase mediates tissue factordependent surface procoagulant activity by thrombin in human vascular smooth muscle cells. Circulation 105, 2030-2036 (2002).

33. May AE, Langer $H$, Seizer P, Bigalke B, Lindemann S, Gawaz M. Platelet-leukocyte interactions in inflammation and atherothrombosis. Semin Thromb Hemost 33, 123-127 (2007).

34. M Chatterjee, SNI von Ungern-Sternberg, P Seizer, F Schlegel, M Büttcher, NA Sindhu, S Müller, A Mack and M Gawaz. Platelet-derived CXCL12 regulates monocyte function, survival, differentiation into macrophages and foam cells through differential involvement of CXCR4- Cell Death and Disease (2015) 6, e1989; doi:10.1038/cddis.2015.233.

35. Semple, J. W., J. E. Italiano, Jr., and J. Freedman. Platelets and the immune continuum. Nat. Rev. Immunol. 2011冈11: 264-

36. Kasper, B., E. Brandt, S. Brandau, and F. Petersen. Platelet factor 4 (CXC chemokine ligand 4) differentially regulates respiratory burst, survival, and cytokine expression of human monocytes by using distinct signaling pathways. J. Immunol. 2007, 179: 2584-

37. Soga, F., N. Katoh, T. Inoue, and S. Kishimoto. Serotonin activates human monocytes and prevents apoptosis. J. Invest. Dermatol. 2007, 127: 1947-

38. Rothwell, P.M.;Wilson,M.; Price, J.F.; Belch, J.F.,Meade, T.W.;Mehta, Z. Effect of daily aspirin on risk of cancer metastasis: Astudy of incident cancers during randomised controlled trials. Lancet 2012, 379, $1591-$

39. Guillem-Llobat, P.; Dovizio, M.; Bruno, A.; Ricciotti, E.; Cufino, V.; Sacco, A.; Grande, R.; Alberti, S.; Arena, V.; Cirillo, M.; et al. Aspirin prevents colorectal cancer metastasis in mice by splitting the crosstalk between platelets and tumor cells. Oncotarget 2016, 7, 32462-32477.

40. Tamar Tadmor, MD; Alessia Bari, MD; Luigi Marcheselli, MS; Stefano Sacchi, MD;Ariel Aviv, MD; Luca Baldini, MD; Paolo G. Gobbi, MD; Samantha Pozzi, MD;Paola Ferri, MS; Maria Christina Cox, MD; Nicola Cascavilla, MD;Emilio lannitto, MD; Massimo Federico, MD; and Aaron Polliack, MD.Absolute Monocyte Count and Lymphocyte-Monocyte Ratio Predict Outcome in Nodular Sclerosis Hodgkin Lymphoma: Evaluation Based on Data From 1450 Patients. Mayo Clin Proc. June 2015;90(6):756764, doi.org/10.1016/j.mayocp.2015.03.025

41. T Dosani, F Covut, R Beck, JJ Driscoll, M de Lima and E Malek. Significance of the absolute lymphocyte/monocyte ratio as a prognostic immune biomarker in newly diagnosed multiple myeloma. Blood Cancer Journal (2017) 7, e579; doi:10.1038/bcj.2017.60

42. Luis F. Porrata, David J. Inwards, Stephen M. Ansell, Ivana N. Micallef, Patrick B. Johnston, William J. Hogan, Svetomir N. Markovic. Infused Autograft Lymphocyte to Monocyte Ratio and Survival in Diffuse Large B Cell Lymphoma. Biol Blood Marrow Transplant 20 (2014) 1804-1812

43. Matthew T. Rondina, McKenzie Carlisle, Tamra Fraughton,Samuel M. Brown,Russell R. Miller III, Estelle S. Harris, Andrew S. Weyrich,Guy A. Zimmerman,Mark A. Supiano,and Colin K. Grissom. Platelet-Monocyte Aggregate Formation and Mortality Risk in Older Patients With Severe Sepsis and 
Septic Shock. J Gerontol A Biol Sci Med Sci. 2015 February;70(2):225-231 doi:10.1093/gerona/glu082.

44. Adam J. Białas, Kamil Kornicki, Maciej Ciebiada囚Adam Antczak, Przemysław Sitarek, Joanna Miłkowska-Dymanowska, Wojciech J. Piotrowski, Paweł Gó Monocyte to large platelet ratio as a diagnostic tool for pulmonary embolism in patients with acute exacerbation of chronic obstructive pulmonary disease. Pol Arch Intern Med. 2018;128 (1): 15-23,doi:10.20 452/pamw.4141

\section{Tables}

Due to technical limitations, table 1 to 5 is only available as a download in the Supplemental Files section.

\section{Figures}

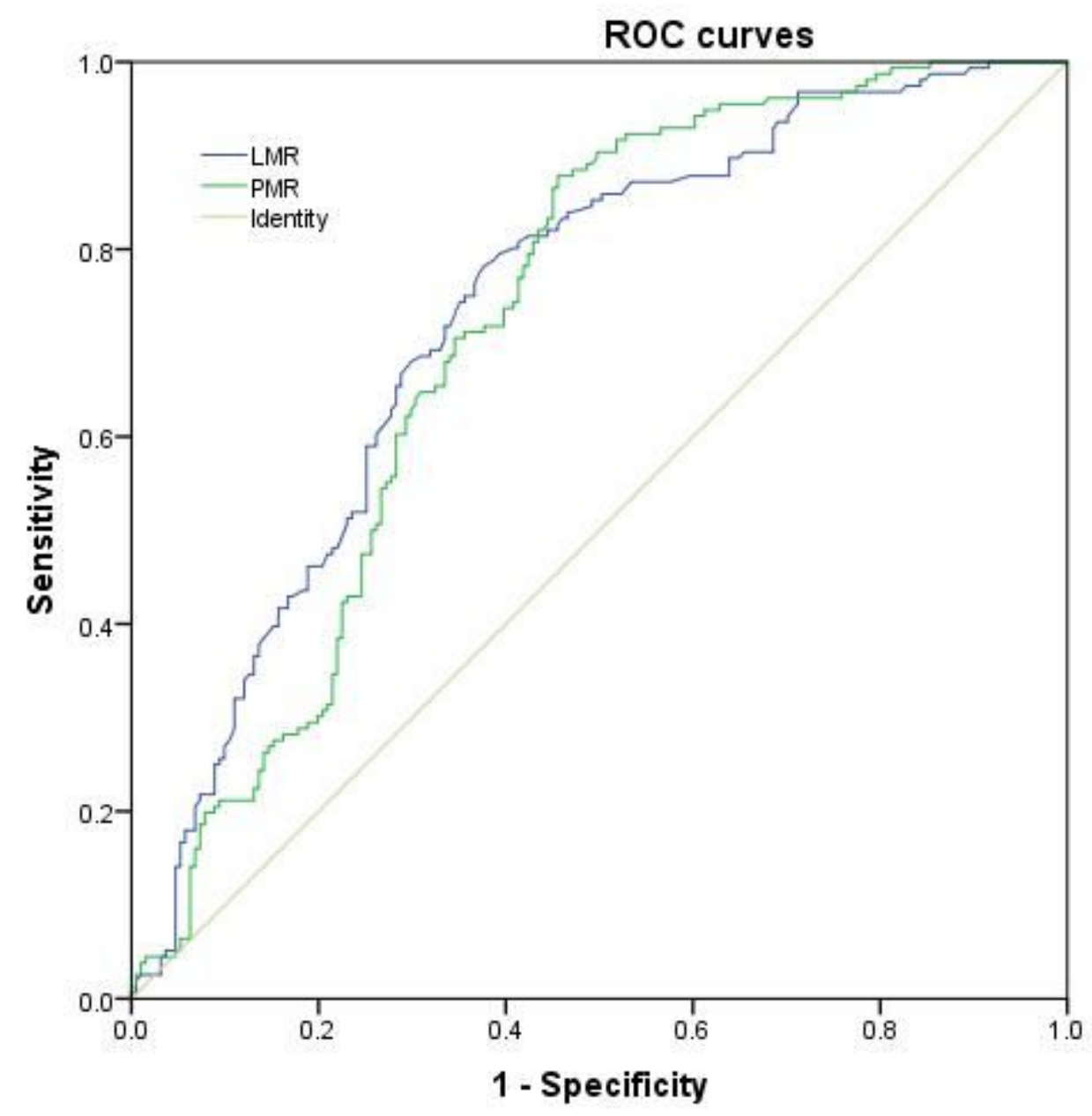

Figure 1

Receiver operating characteristic (ROC) curves of LMR and PMR of patients with PTCL. The AUC of LMR and PMR were 0.734 (95\% Cl: 0.682-0.786), 0.718(95\% Cl: 0.664-0.772). 
(A)

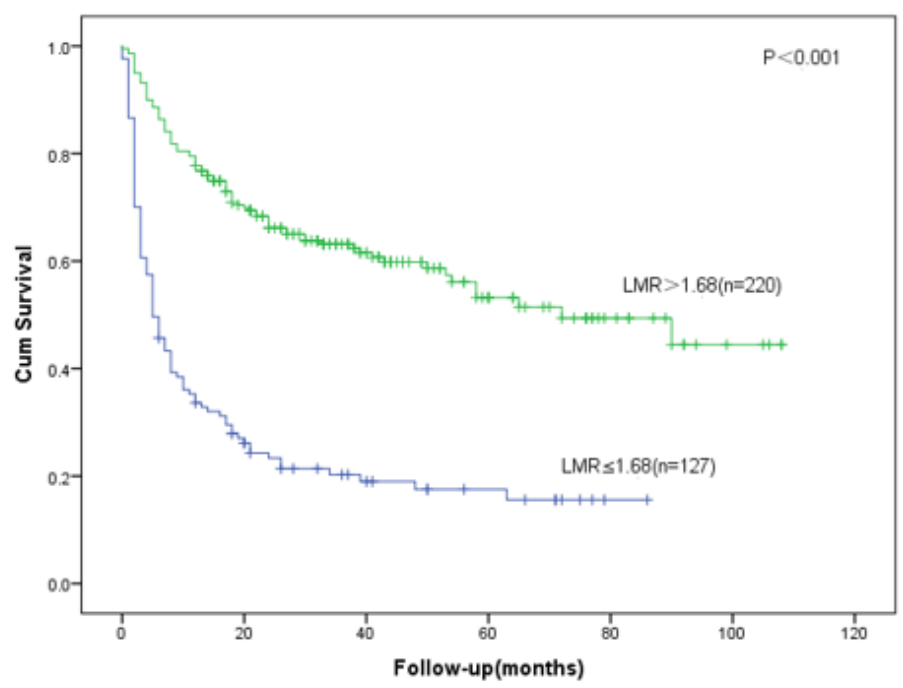

(B)

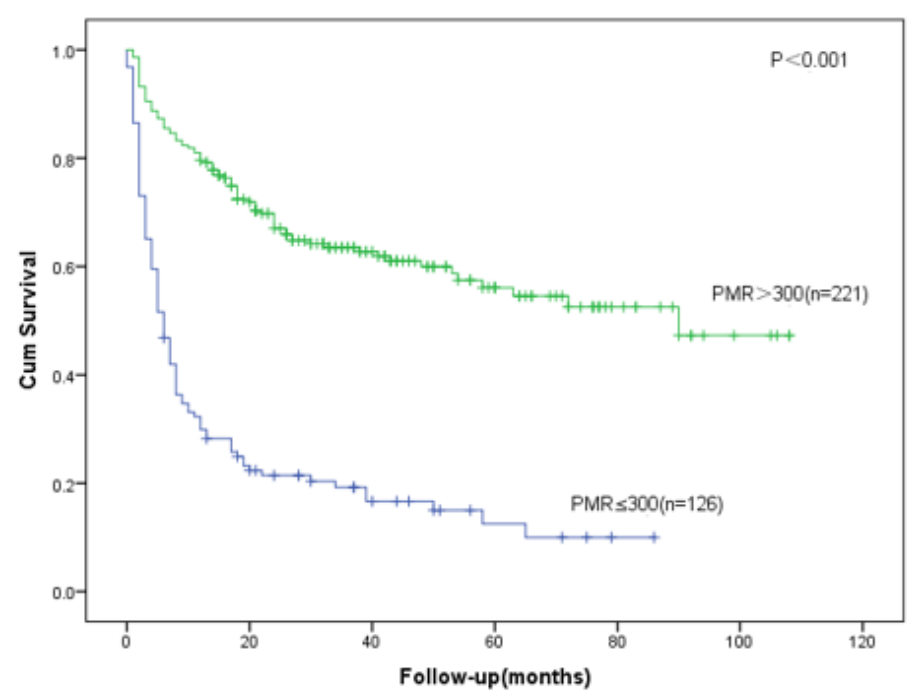

Figure 2

OS: Overall survival. (A) OS for different levels of LMR; (B) OS for different levels of PMR. 
(A)

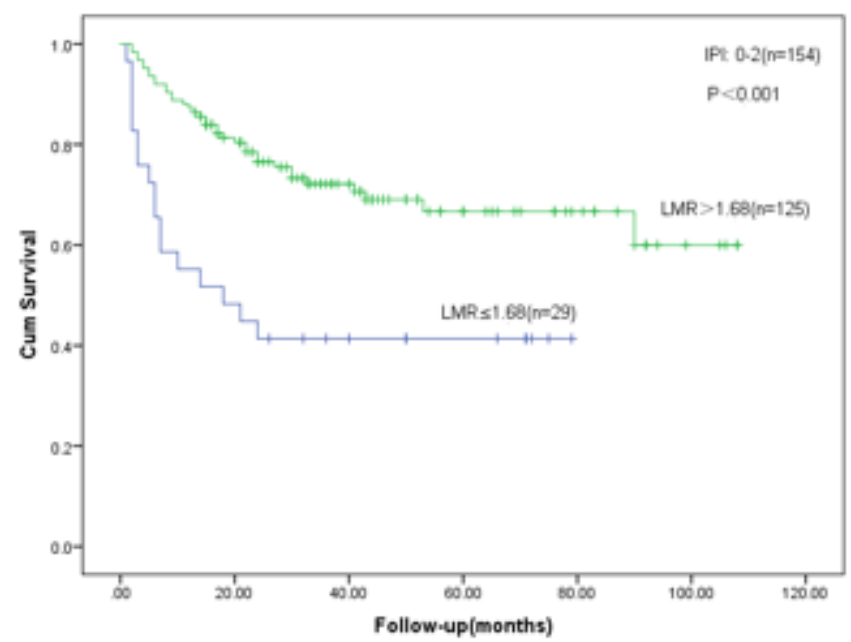

(C)

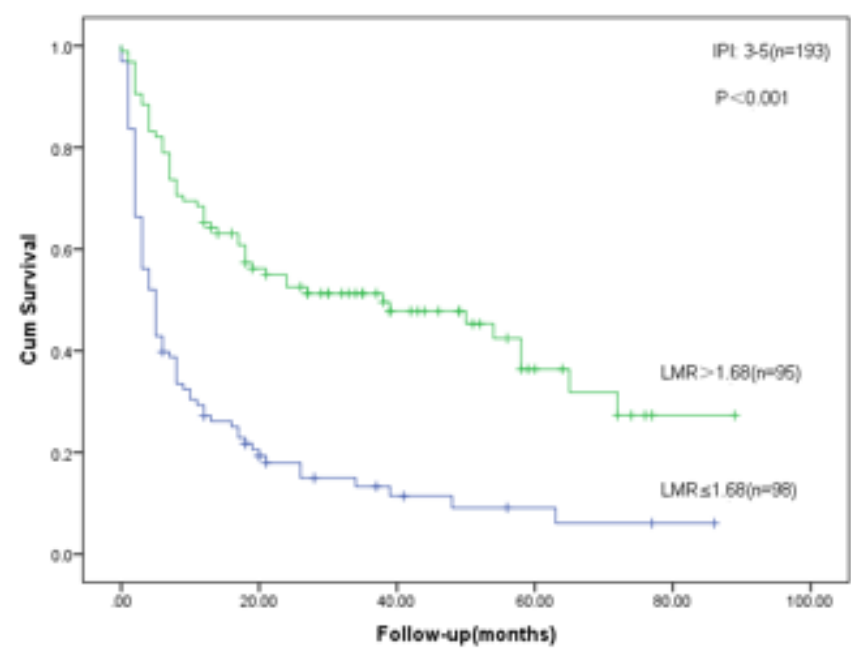

(B)

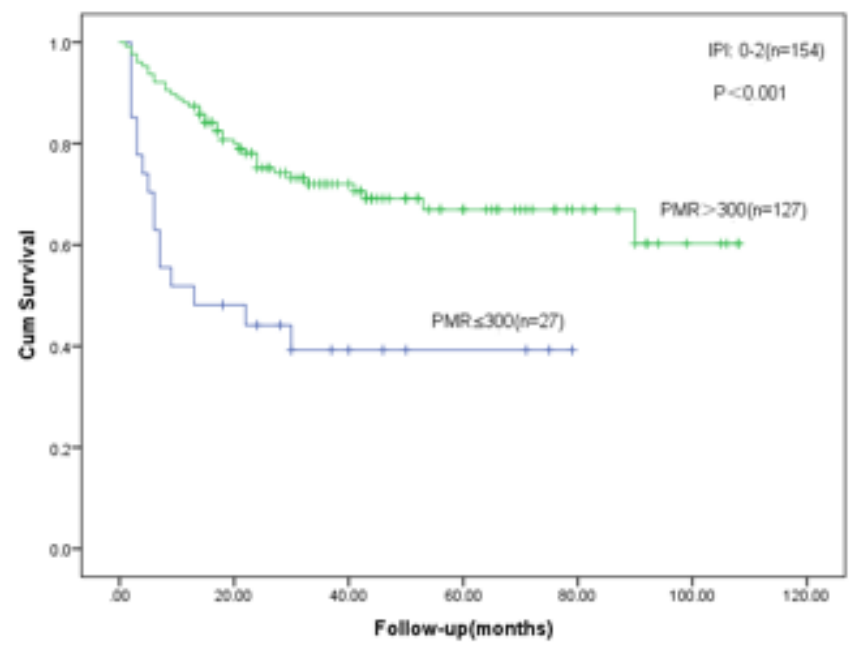

(D)

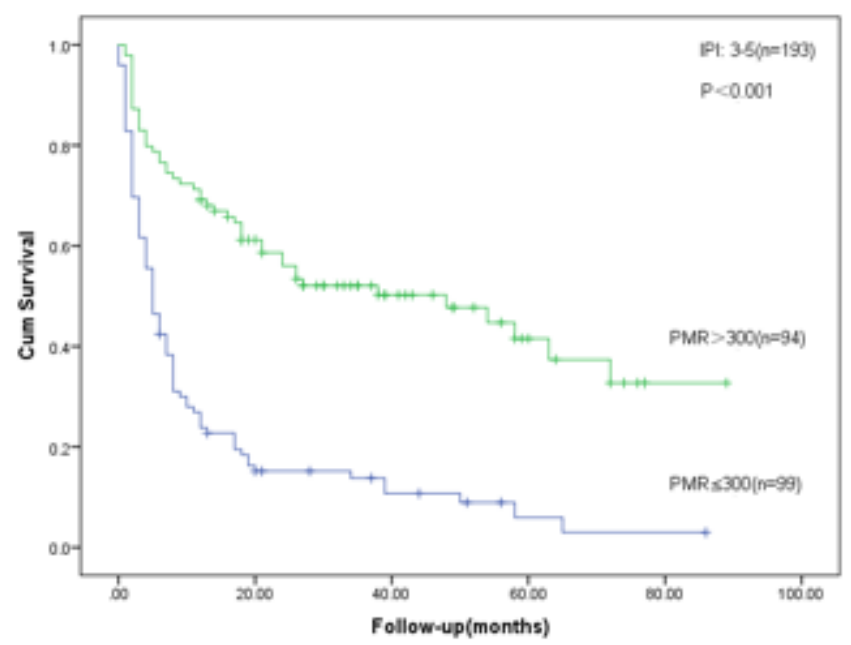

Figure 3

OS for different IPI risk stratification of PTCL patients. (A)OS of patients with different LMR levels in group of IPI score 0-2; (B)OS of patients with different PMR levels in group of IPI score 0-2; (C)OS of patients with different LMR levels in group of IPI score 3-5; (D)OS of patients with different PMR levels in group of IPI score 3-5. 


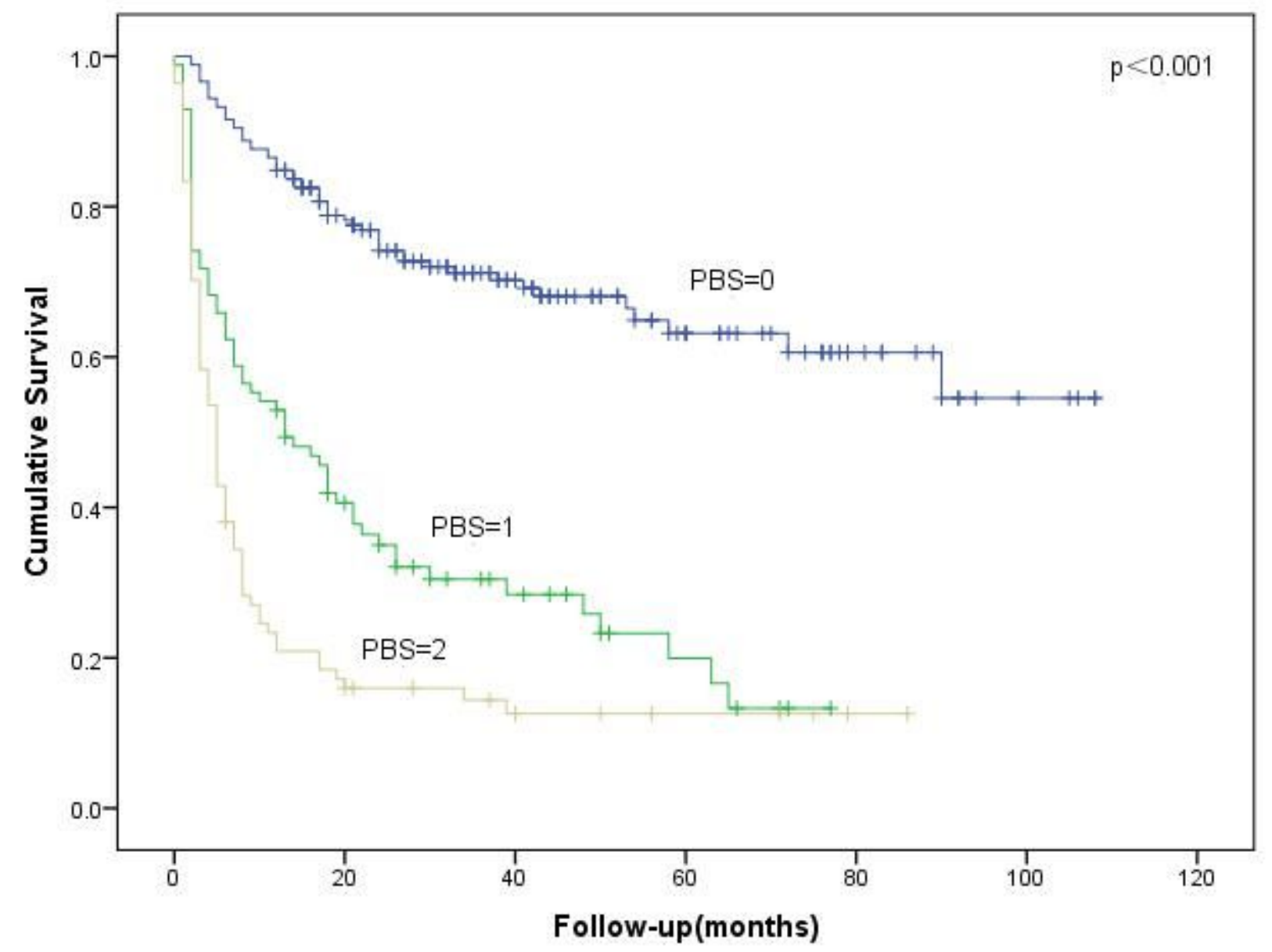

Figure 4

Prognostic PBS model in OS of Peripheral T-cell lymphoma. Abbreviation: PBS, Peripheral Blood Score.

\section{Supplementary Files}

This is a list of supplementary files associated with this preprint. Click to download.

- table1.docx

- table2.doc

- table3.doc

- table4.doc

- table5.doc 